\title{
Control of Nanoscale Friction on Gold in an Ionic Liquid by a Potential-Dependent Ionic Lubricant Layer
}

\author{
James Sweeney, ${ }^{1}$ Florian Hausen, ${ }^{2}$ Robert Hayes, ${ }^{1}$ Grant B. Webber, ${ }^{1}$ Frank Endres, ${ }^{3}$ Mark W. Rutland, ${ }^{4}$ \\ Roland Bennewitz, ${ }^{2}$ and Rob Atkin ${ }^{1, *}$ \\ ${ }^{1}$ Centre for Advanced Particle Processing and Transport, The University of Newcastle, Callaghan, NSW 2308, Australia \\ ${ }^{2}$ INM-Leibniz-Institute for New Materials, Campus D2 2, 66123 Saarbrücken, Germany \\ ${ }^{3}$ Institute of Particle Technology, Clausthal University of Technology, Arnold-Sommerfeld-Strasse 6, \\ 38678 Clausthal-Zellerfeld, Germany \\ ${ }^{4}$ Department of Chemistry, Surface and Corrosion Science, Royal Institute of Technology, Drottning Kristinas Väg 51, \\ SE-100 44 Stockholm, Sweden and YKI, Institute for Surface Chemistry, Box 5607, 11486 Stockholm, Sweden
}

(Received 6 June 2012; published 10 October 2012)

\begin{abstract}
The lubricating properties of an ionic liquid on gold surfaces can be controlled through application of an electric potential to the sliding contact. A nanotribology approach has been used to study the frictional behavior of 1-butyl-1-methylpyrrolidinium tris(pentafluoroethyl) trifluorophosphate ([ $\left.\left.\mathrm{Py}_{1,4}\right] \mathrm{FAP}\right)$ confined between silica colloid probes or sharp silica tips and a $\mathrm{Au}(111)$ substrate using atomic force microscopy. Friction forces vary with potential because the composition of a confined ion layer between the two surfaces changes from cation-enriched (at negative potentials) to anion-enriched (at positive potentials). This offers a new approach to tuning frictional forces reversibly at the molecular level without changing the substrates, employing a self-replenishing boundary lubricant of low vapor pressure.
\end{abstract}

DOI: 10.1103/PhysRevLett.109.155502

PACS numbers: $62.20 . \mathrm{Qp}$

Much scientific interest is aimed at understanding the interfacial forces and processes that occur between two surfaces in contact [1-3]. Tribological effects, which include friction, adhesion, and wear, are ubiquitous when any two surfaces are brought together and can be measured across all length and time scales [4-6].

Room-temperature ionic liquids (ILs) are a subset of molten salts, distinguished by having melting points below room temperature. Their low melting points are brought about by both weakening the electrostatic interactions between the ions and hindering their packing into a crystal lattice. Electrostatic forces are reduced by engineering the molecular structure so that at least one of the ions is large and organic, which increases the distance between neighboring charged centers, and by delocalizing the ionic charge over a large molecular volume. Crystal packing is hindered by introducing asymmetry in at least one of the ions. ILs often have wide temperature and redox stability ranges, and some of them can be classified as green solvents on account of their vanishingly low vapor pressures, especially if made from biocompatible sources.

At the nanoscale, ILs display richer ordering than conventional liquids, and many form well-defined nanostructures both in the bulk phase [7-9] and at interfaces [10-16]. This arises from the combination of long- (Coulombic) and short-range (van der Waals, hydrogen bonding, dipoledipole, and solvophobic [17]) interactions present between ions and in many respects is reminiscent of self-assembly in aqueous surfactant mesophases but with dimensions at least an order of magnitude smaller [13].
The interfacial properties of ILs mean they have great potential as lubricants. Near charged [10-16] and uncharged [11,13] solid surfaces, ILs can rearrange into more ordered layers reminiscent of lamellae, with thickness consistent with the size of an ion pair [10]. As the first ion layer interacts strongly with oppositely charged surfaces [13], ILs resist "squeeze-out" as surfaces are compressed, resulting in a film that remains in place at higher forces than for a comparable molecular lubricant [18]. Moreover, the sterically mismatched ion shapes hinder attractive interactions between liquid layers, preventing stiction and allowing the layers to slide easily over one another. While a significant number of papers have examined IL lubrication macroscopically [19,20], there are few nanotribology reports aimed at elucidating the molecular origin of IL lubrication. Here, we systematically study the nanometer-scale friction as a function of surface potential, which controls the ionic composition of the ion layer at the charged interface. Experiments were performed in two different atomic force microscopy (AFM) labs, using sharp AFM tips (estimated tip radii $10-25 \mathrm{~nm}$ ) or colloid probes attached to V-shaped AFM cantilevers (probe diameter $5 \mu \mathrm{m}$ ). The same ultrapure IL, 1-butyl-1-methylpyrrolidinium tris(pentafluoroethyl) trifluorophosphate $\left(\left[\mathrm{Py}_{1,4}\right] \mathrm{FAP}\right)$ (all impurities lower than $10 \mathrm{ppm}$ ), was used by both groups.

Force measurements using sharp AFM tips were performed using an Agilent 5500 AFM and following the experimental procedures for sample preparation and measurements described in Ref. [21], except that a platinum wire quasireference electrode was employed. 
Force measurements using colloid probes were acquired by a Digital Instruments NanoScope IV Multimode AFM with an E scanner in contact mode. Experimental details, including the procedure used to construct the AFM electrochemical cell, cleaning of components, etc., are exactly as described in Ref. [14], except that a silica colloid probe (5 $\mu \mathrm{m}$ diameter, Bangs Laboratories, Inc.) was attached to the tip of the $\mathrm{Si}_{3} \mathrm{~N}_{4}$ cantilever (Digital Instruments, CA) [22]. Friction was measured by scanning perpendicular to the cantilever's long axis with the slow scan axis disabled. The scan size and scan speed were $500 \mathrm{~nm}$ and $6.0 \mu \mathrm{m} \cdot \mathrm{s}^{-1}$, respectively. The lateral deflection signal (i.e., cantilever twist) was converted to lateral force using a custom-built function produced in MATLAB 7.11.0 which takes into account the torsional spring constant and the geometry of the cantilever.

The electrochemical modification of surfaces can be followed by cyclic voltammetry. In order to reveal the influence of such modifications on friction, we have simultaneously recorded electrochemical current and friction forces as a function of potential in the AFM electrochemical cell. Figure 1 demonstrates a significant change in friction, where friction increases strongly with potential in the range from -1.75 to $-0.5 \mathrm{~V}$. The cyclic voltammogram, although of lower resolution than those recorded in dedicated electrochemical experiments, exhibits all characteristic peaks reported for $\left[\mathrm{Py}_{1,4}\right] \mathrm{FAP}$ and $\mathrm{Au}(111)$ $[14,16]$. The inset in Fig. 1 shows a lateral force map recorded at a potential of $-1.3 \mathrm{~V}$. It is composed of atomic stick-slip events with a sawtooth-shaped characteristic,

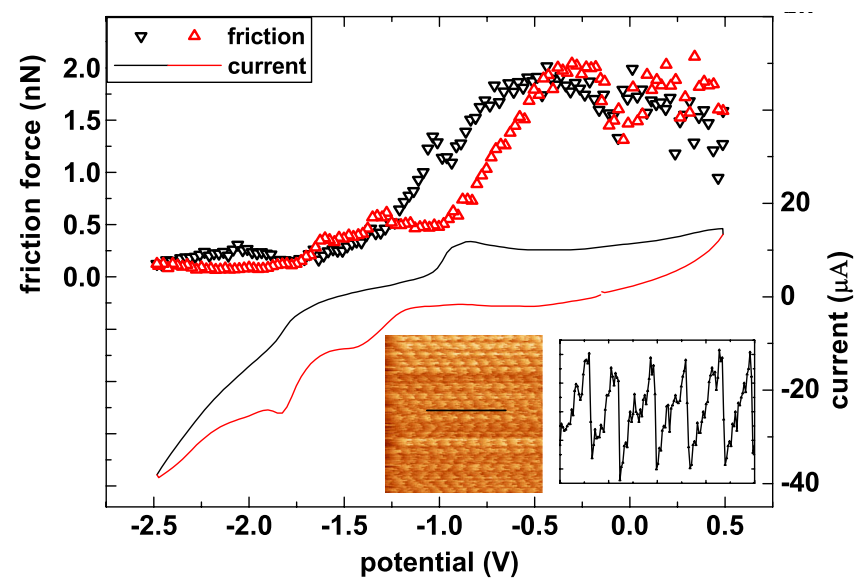

FIG. 1 (color online). Cyclic voltammogram and simultaneously recorded friction force for a sharp AFM tip sliding on $\mathrm{Au}(111)$ in $\left[\mathrm{Py}_{1,4}\right] \mathrm{FAP}$ [load $1.5 \mathrm{nN}$, potential sweep rate $15 \mathrm{mV} \cdot \mathrm{s}^{-1}$; black (dark) triangles indicate increasing and red (lighter) triangles decreasing potential]. The inset is a lateral force map (3.8 nm side length) showing the periodicity of the $\mathrm{Au}(111)$ surface, recorded at a potential of $-1.3 \mathrm{~V}$ after approaching the tip to the gold surface. The inset graph shows lateral forces between -0.25 and $0.25 \mathrm{nN}$ along a distance of $2 \mathrm{~nm}$, indicated by the bar in the lateral force map. which follow the periodicity of the $\mathrm{Au}(111)$ surface. At this potential, the tip can penetrate all ion layers into contact with the $\mathrm{Au}(111)$ surface, as revealed in force versus distance profiles [14,23] and already indicated by the successful imaging of the reconstructed $\mathrm{Au}(111)$ surface in scanning tunneling microscopy [16].

The tribological effects of the applied potential are confirmed by the friction results for colloid probes shown in Fig. 2. Friction force is recorded as a function of applied load and potential for increasing normal loads up to $25 \mathrm{nN}$. For all potentials, the lateral force increases with normal load, but the rate of increase-i.e., the friction coefficient-depends on the surface potential, with higher coefficients measured at more positive potentials. These experiments were repeated on five separate occasions, using fresh IL and $\mathrm{Au}(111)$ substrates each time and three different colloid probes. While the magnitude of the lateral forces did vary between experiments, the trend in friction force with potential was always the same. Data consistent with this trend were also obtained at $\pm 0.5 \mathrm{~V}$ but have not been included in Fig. 2 for clarity of presentation. The level of variability in the friction data, most notable in the experiments at potentials of -1.0 and $-2.0 \mathrm{~V}$, was also consistent between experiments.

The composition of the interfacial layer as a function of potential plays a key role in determining lubricity. Note that a significant contribution of adhesion to friction can be excluded as frictional forces tend towards zero for vanishing load. Normal force vs distance curves recorded with the colloid probe at the potentials used in Fig. 2 show that the probe displaces ordered layers of IL ion pairs. For positive potentials, the probe displaces the last cation layer for normal loads greater than $2.5 \mathrm{nN}$ but never displaces the last anion layer for all normal loads used in these experiments. Correspondingly, for negative potentials, the probe displaces the last anion layer but at no force displaces the cation layer. Force profiles and details of this analysis can be found in the Supplemental Material [23]. We limit our discussion of the results presented in Fig. 2 to normal loads greater than $5 \mathrm{nN}$, where clear differences in lubricity are evident as the potential is changed. We attribute these differences to the composition of a single layer of ions confined between the silica colloid probe and the $\mathrm{Au}(111)$ surface.

The lateral forces measured for -1 and $-2 \mathrm{~V}$ are the same within error. At these negative potentials, the interfacial layer is cation rich. Because the cations are amphiphilic, their conformation can vary with applied potential [14,24], but the most likely ion orientation is with the charged ammonium headgroup in contact with the $\mathrm{Au}(111)$ surface and alkyl chains pointing away from the interface. Some portion of the cations may be oriented with charged groups facing the silica surface, but at these potentials this proportion is expected to be low. Small lateral forces and low friction coefficients $(0.20$ for $-1 \mathrm{~V}$ and 0.19 


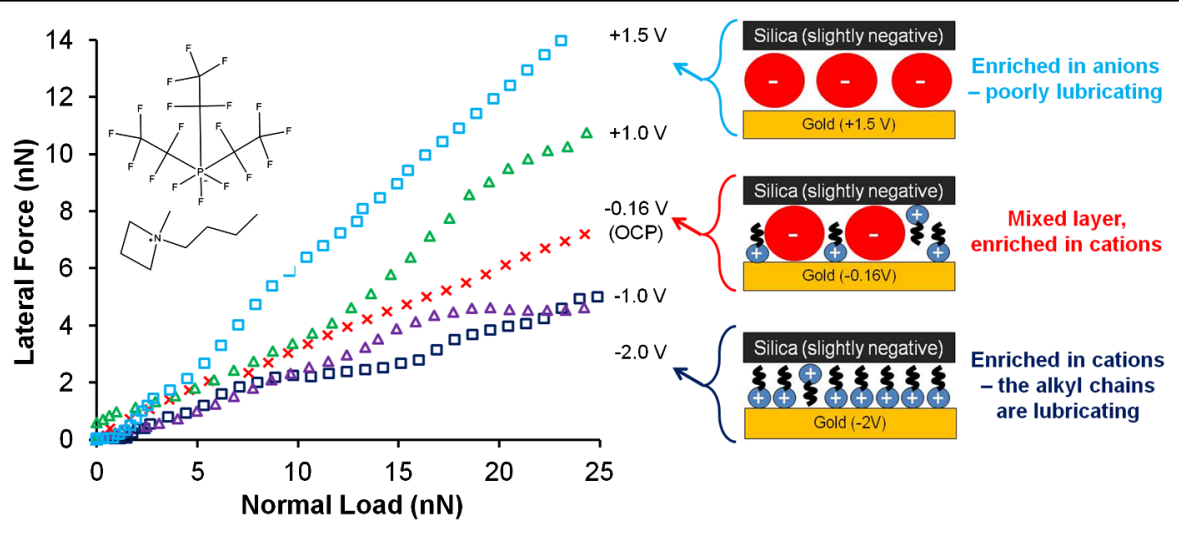

FIG. 2 (color online). Lateral force versus normal load for different surface potentials for $\left[\mathrm{Py}_{1,4}\right]$ FAP (see ion pair inset) confined between a silica colloid probe and the $\mathrm{Au}(111)$ electrode surface. The potential for each data set is as indicated in the figure (scan size $500 \mathrm{~nm}$, scan speed $6.0 \mu \mathrm{m} \cdot \mathrm{s}^{-1}$ ). Each individual lateral force data point is an average of at least six repeated friction loops to produce statistically significant data.

for $-2 \mathrm{~V}$ ) are measured at these potentials because the probe slides along a well-defined plane of dense alkyl chains. This is analogous to nanotribology mechanisms reported for long-chain oils [25] or self-assembled monolayers [26] that brush past each other under confinement. The similarity of the -1 and $-2 \mathrm{~V}$ data in Fig. 2 suggests there is little change in either the interfacial layer composition or ion orientation under the colloid probe as potential is decreased from -1 to $-2 \mathrm{~V}$.

At open circuit potential, the potential of the $\mathrm{Au}(111)$ surface is slightly negative at $-0.16 \mathrm{~V}$ versus $\mathrm{Pt}$, and the silica surface is also weakly negatively charged $[11,15]$. The interfacial layer in this system is thus of mixed composition [15] but slightly enriched in the cation, which could be oriented towards either surface. Lateral forces are increased compared to those obtained at -1 and $-2 \mathrm{~V}$, leading to a higher friction coefficient (0.28). This shows that $\mathrm{FAP}^{-}$ions in the interfacial layer decrease lubricity. This is likely due to an increased molecular roughness arising from the disparity in size of the $\mathrm{FAP}^{-}$anions and the $\left[\mathrm{Py}_{1,4}\right]^{+}$cations (cf. the ion pair inset in Fig. 2), which will lead to a relatively poorly defined sliding plane. Conformational changes in the mixed layer may also lead to additional opportunities to dissipate energy.

When the potential is raised further to more positive potentials, the effect of the anion becomes more pronounced. At positive potentials, the cation is electrostatically repelled from the surface, and the interfacial layer is enriched in the anion. The friction coefficient is now quite high at $0.45(+1 \mathrm{~V})$ and $0.59(+1.5 \mathrm{~V})$, indicating poor lubricity at these potentials. The fact that lateral forces are greater at $1.5 \mathrm{~V}$ than at $1 \mathrm{~V}$ suggests that some cations are present in the interfacial layer at $1 \mathrm{~V}$, but their number decreases at $1.5 \mathrm{~V}$.

There are a number of possible reasons for the lubricating action of cations. For instance, surface bound FAP ${ }^{-}$ anions have a disordered coil morphology [27], which is less lubricating than the exposed alkyl tails of surface bound cations, resulting in higher friction. Alternatively, molecular factors (increased size, charge delocalization) of the $\mathrm{FAP}^{-}$species mean that anions are more easily stripped off the surface than cations, leading to local solid-solid contacts that correspond to high friction.

The results for the sharp AFM tip presented in Fig. 1 support the picture developed above of a different lubricity of cation and anion layers. At potentials of $-1.5 \mathrm{~V}$ and below, not even the sharp AFM tip can displace the last cation layer (see the Supplemental Material [23]). This cation layer provides a reliable and stable low-friction configuration. In a small potential window around $-1.3 \mathrm{~V}$, both the last cation and anion layers are displaced and atomic friction of the $\mathrm{Au}(111)$ surface is observed at slightly higher friction values. So far, we have not observed the molecular structure of either the gold surface or the bound layers at other potentials; such imaging has been shown to be possible in other systems, for example, on specifically adsorbed sulfate ions in aqueous electrolytes [21]. With increasing potential, the increasingly mixed layer of cations and anions results in increased friction with significant variability, which is probably caused by the interaction of the sharp AFM tip with an inhomogeneous and less ordered layer of ions. As a consequence, the $\left[\mathrm{Py}_{1,4}\right]$ FAP allows the control of friction by applied potential while avoiding the structural changes of an oxide formation, which were observed for aqueous electrolytes [28].

Our results open the way to a method of switching friction by switching between anion and cation layers in ILs, as controlled by the potential applied to the substrate surface. This opportunity is further explored in an experiment using the sharp AFM tip, which is summarized in Fig. 3. For increasing values of the normal load, the potential is switched between the anion layer and the cation layer at each load. The resulting friction values reproduce the key result reported for the colloid probe in Fig. 2. The friction coefficient measured for the cation layer is significantly lower than for the anion layer in the case of the sharp 


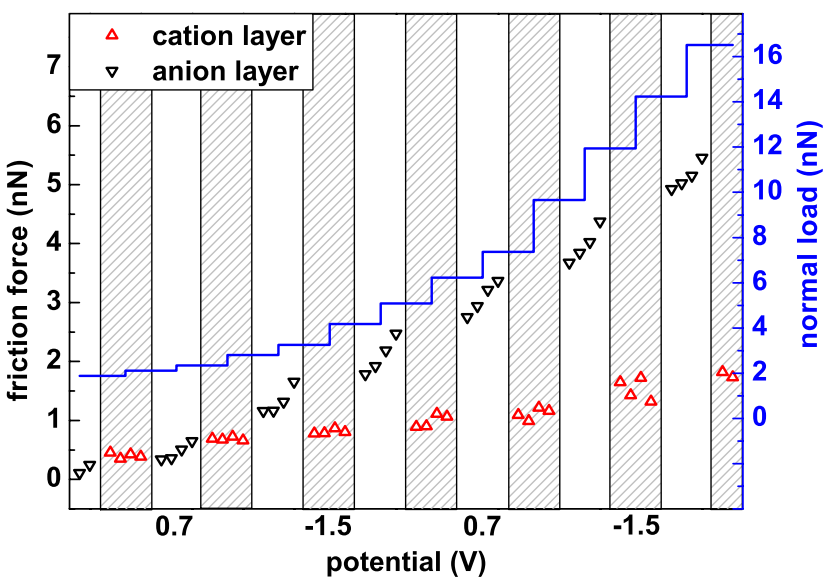

FIG. 3 (color online). Friction forces as functions of load and applied potential recorded for a sharp AFM tip sliding on $\mathrm{Au}(111)$ in $\left[\mathrm{Py}_{1,4}\right] \mathrm{FAP}$. Each data point represents the average over a full scan frame of $14 \mathrm{~nm}$ side length. The potential is switched between -1.5 and $0.7 \mathrm{~V}$ for each value of the applied normal load.

AFM tip. Switching friction operates reproducibly at all loads higher than $3 \mathrm{nN}$.

We foresee that the findings reported in this study will impact upon a variety of new important technologies venerable to adhesion, friction, and wear. For example, it is difficult to meet the precise operating standards required over practical lifetimes in micro- and nanoelectromechanical systems because friction must be controlled across tiny dimensions for components with high surface-area-tovolume ratios [29]. Likewise, electrical contacts [30] or surfaces prone to contact electrification [31] stand to benefit, as ILs are electrically conductive lubricants. This latter property is important, as traditional molecular lubricants such as polytetrafluoroethylene are often unsuitable in these situations specifically because they are electrically insulating.

In 2008, Glavatskih and Höglund coined the expression "tribotronics," suggesting active control of bearing performance in industrial machinery through sensors and actuators [32]. This would have the capacity to improve efficiency and reduce wear. Among other things, the authors suggested the possible use of electrorheological fluids. This work demonstrates the potential for (nano) tribotronics-where electric potentials directly control the lubricating properties of a self-replenishing boundary lubricant. The explosion of research interest in ILs over the last decade is largely due to their remarkable physical properties, and the fact that these properties can be controlled systematically through variation in the structure of the ions has led ILs to be referred to as "designer solvents" [13]. The results presented in this Letter show that electrode potential can be used to control lubricity and thus provides a new, hitherto overlooked, IL design parameter.
This research was supported by ARC Discovery Projects (No. DP0986194 and No. DP120102708) and by Deutsche Forschungsgemeinschaft (DFG) within the Priority Program SPP 1191-Ionic Liquids. The Newcastle group thanks Associate Professor Scott Donne for the loan of the potentiostat. R. H. thanks AINSE for a PGRA. M. R. acknowledges support from the Swedish Research Council (VR) and the Swedish Strategic Research Foundation. F. H. and R. B thank Eduard Arzt for continuous support of this project. J.S., F. H., and R. H. contributed equally to the work described in this Letter.

*Corresponding author

Rob.Atkin@newcastle.edu.au

[1] F. P. Bowden and D. Tabor, The Friction and Lubrication of Solids (Oxford University Press, Oxford, England, 2001).

[2] B. Bhushan, Handbook of Micro/Nanotribology (CRC Press, Boca Raton, FL, 1999).

[3] J. N. Israelachvili, Intermolecular and Surface Forces (Elsevier, London, 2011).

[4] C. M. Mate, G. M. McClelland, R. Erlandsson, and S. Chiang, Phys. Rev. Lett. 59, 1942 (1987).

[5] C. H. Scholz, Nature (London) 391, 37 (1998).

[6] R. Budakian, K. Weninger, R. A. Hiller, and S. J. Putterman, Nature (London) 391, 266 (1998).

[7] A. Triolo, O. Russina, H.-J. Bleif, and E. Di Cola, J. Phys. Chem. B 111, 4641 (2007).

[8] R. Atkin and G. G. Warr, J. Phys. Chem. B 112, 4164 (2008).

[9] R. Hayes, S. Imberti, G. G. Warr, and R. Atkin, Phys. Chem. Chem. Phys. 13, 3237 (2011).

[10] R. G. Horn, D. F. Evans, and B. W. Ninham, J. Phys. Chem. 92, 3531 (1988).

[11] R. Atkin and G. G. Warr, J. Phys. Chem. C 111, 5162 (2007).

[12] M. Mezger et al., Science 322, 424 (2008).

[13] R. Hayes, G. G. Warr, and R. Atkin, Phys. Chem. Chem. Phys. 12, 1709 (2010).

[14] R. Hayes, N. Borisenko, M. K. Tam, P. C. Howlett, F. Endres, and R. Atkin, J. Phys. Chem. C 115, 6855 (2011).

[15] K. Shimizu, A. Pensado, P. Malfreyt, A. A. H. Pádua, and J. N. Canongia Lopes, Faraday Discuss. 154, 155 (2012).

[16] R. Atkin, N. Borisenko, M. Drüschler, S. Z. El Abedin, F. Endres, R. Hayes, B. Huber, and B. Roling, Phys. Chem. Chem. Phys. 13, 6849 (2011).

[17] A. Ray, Nature (London) 231, 313 (1971).

[18] S. Perkin, T. Albrecht, and J. Klein, Phys. Chem. Chem. Phys. 12, 1243 (2010).

[19] I. Minami, Molecules 14, 2286 (2009).

[20] F. U. Shah, S. Glavatskih, D. R. MacFarlane, A. Somers, M. Forsyth, and O.N. Antzutkin, Phys. Chem. Chem. Phys. 13, 12865 (2011).

[21] F. Hausen, N. N. Gosvami, and R. Bennewitz, Electrochim. Acta 56, 10694 (2011). 
[22] W. A. Ducker, T. J. Senden, and R. M. Pashley, Nature (London) 353, 239 (1991).

[23] See Supplemental Material at http://link.aps.org/ supplemental/10.1103/PhysRevLett.109.155502 for this document.

[24] S. Baldelli, Acc. Chem. Res. 41, 421 (2008).

[25] C. Drummond, N. Alcantar, and J. Israelachvili, Phys. Rev. E 66, 011705 (2002).

[26] N. J. Brewer, B. D. Beake, and G. J. Leggett, Langmuir 17, 1970 (2001).
[27] T. Waldmann, H.-H. Huang, H. E. Hoster, O. Höfft, F. Endres, and R. J. Behm, Chem. Phys. Chem. 12, 2565 (2011).

[28] A. Labuda, F. Hausen, N. N. Gosvami, P. H. Grutter, R. B. Lennox, and R. Bennewitz, Langmuir 27, 2561 (2011).

[29] M. Palacio and B. Bhushan, Adv. Mater. 20, 1194 (2008).

[30] W. E. Cambell, IEEE Trans. Components Hybrids Manuf. Technol. 1, 4 (1978).

[31] R. G. Horn and G. D. Smith, Science 256, 362 (1992).

[32] S. Glavatskih and E. Höglund, Tribol. Int. 41, 934 (2008). 